\title{
Vacuolar H+-ATPase as target to restore cardiac function in the diabetic heart
}

Citation for published version (APA):

Wang, S. (2020). Vacuolar H+-ATPase as target to restore cardiac function in the diabetic heart. [Doctoral Thesis, Maastricht University]. Gildeprint Drukkerijen. https://doi.org/10.26481/dis.20200908sw

Document status and date:

Published: 01/01/2020

DOI:

10.26481/dis.20200908sw

Document Version:

Publisher's PDF, also known as Version of record

\section{Please check the document version of this publication:}

- A submitted manuscript is the version of the article upon submission and before peer-review. There can be important differences between the submitted version and the official published version of record.

People interested in the research are advised to contact the author for the final version of the publication, or visit the DOI to the publisher's website.

- The final author version and the galley proof are versions of the publication after peer review.

- The final published version features the final layout of the paper including the volume, issue and page numbers.

Link to publication

\footnotetext{
General rights rights.

- You may freely distribute the URL identifying the publication in the public portal. please follow below link for the End User Agreement:

www.umlib.nl/taverne-license

Take down policy

If you believe that this document breaches copyright please contact us at:

repository@maastrichtuniversity.nl

providing details and we will investigate your claim.
}

Copyright and moral rights for the publications made accessible in the public portal are retained by the authors and/or other copyright owners and it is a condition of accessing publications that users recognise and abide by the legal requirements associated with these

- Users may download and print one copy of any publication from the public portal for the purpose of private study or research.

- You may not further distribute the material or use it for any profit-making activity or commercial gain

If the publication is distributed under the terms of Article $25 \mathrm{fa}$ of the Dutch Copyright Act, indicated by the "Taverne" license above, 


\section{Summary}

Under normal physiological circumstances, the heart is a metabolically flexible organ that can utilize a wide range of substrates for energy provision, and therefore can be regarded as an omnivore. To meet it's immediate energy needs, the healthy adult heart mainly uses longchain fatty acids (FA) and glucose, whereas it also may shift its energy substrate preference to alternative substrates like amino acids (AA) and ketone bodies when being exposed to physiological or pathological stimuli. Recent studies from our lab have shown that vacuolartype $\mathrm{H}^{+}$-ATPase (v-ATPase) is closely linked to cardiac energy metabolism. V-ATPase, a multimeric protein complex (>14 subunits), is structurally divided into a cytosolic $\mathrm{V}_{1}$ subcomplex and a transmembrane $\mathrm{V}_{0}$ sub-complex, encompassing the ATP catalyzing activity and a rotary mechanism so as to maintain endosomal acidification, respectively. Notably, assembly/disassembly cycles of the $\mathrm{V}_{1}$ and $\mathrm{V}_{0}$ sub-complexes is rapid and reversible, so that it leads to rapid modulation of v-ATPase function. As earlier established, upon disassembly of the two sub-complexes, such as when cells are subjected to lipid oversupply or glucose withdrawal, v-ATPase function declines. Accordingly, the general aim of this thesis was to test the potential roles of cardiac energy substrates, i.e., lipid (palmitate), glucose, amino acids (AA), and ketone bodies, on the functioning of v-ATPase in the heart, either directly or indirectly. Moreover, we also investigated whether the possible roles of v-ATPase re-assembly are regulated by these energy substrates in lipid-induced insulin resistance and contractile dysfunction, with focus on unraveling the underlying molecular mechanism.

Chapter 1 provides a general introduction on the supply and utilization of the energy substrates lipid (palmitate), glucose, AA, and ketone bodies by (rodent/human) cardiomyocytes. Additionally, this chapter also describes the roles of FA transporter CD36 and glucose transporter GLUT4 in cardiac lipid accumulation, and then explains the possible mechanism by which lipids impair the function of v-ATPase thereby setting the heart on a road towards diabetic cardiomyopathy. At the end of this chapter, the aim and the outline of this thesis has been further discussed in greater detail.

Chapter 2 highlights that increased cellular glucose availability rescues lipid-induced vATPase impairment in rodent/human cardiomyocytes. Specifically, this restoration of vATPase function leads to endosomal acidification in lipid-overloaded (rodent/human) cardiomyocytes, thus resulting in a decrease of CD36-mediated lipid accumulation which, in turn, improves insulin-stimulated glucose uptake as well as contractile function. As a result, 
this chapter provides the first proof-of-principle for testing potential strategies to counteract lipid-induced insulin resistance and contractile dysfunction via v-ATPase re-assembly.

Chapter 3 describes observations indicating that in (rodent/human) cardiomyocytes, addition of AA, in particular a mixture of arginine/leucine/lysine, stimulates mTOR activation, which then binds to the $\mathrm{V}_{1}$ subcomplex of v-ATPase, thereby facilitating $\mathrm{V}_{1}$ part to re-assemble with $\mathrm{V}_{0}$. Given that v-ATPase is a scaffold for mTOR activation, this chapter proposes that mTOR activation also induces v-ATPase activation, suggesting that the relationship between $\mathrm{v}$-ATPase and mTOR operates in both directions. Moreover, this AA-activated mTOR-vATPase axis leads to endosomal acidification, and thus inhibits CD36-mediated FA uptake to protect cardiomyocytes from lipid overload-induced insulin resistance and contractile dysfunction. Hence, the data presented in this chapter suggest that this specific AA-induced mechanism through v-ATPase re-assembly may offer simple and effective treatment options to combat lipid-induced cardiomyopathy.

Chapter 4 describes that chronic exposure of (rodent/human) cardiomyocytes to the ketone body 3 - $\beta$-hydroxybutyrate (3HB) results in v-ATPase disassembly, and subsequently into loss of v-ATPase activity. Similarly to lipid exposure, 3HB-induced v-ATPase impairment results in the loss of endosomal acidification, followed by CD36 translocation to the sarcolemma, myocellular lipid accumulation, insulin resistance and finally contractile dysfunction, whereas all these maladaptive alterations were completely prevented by addition of the specific AA (a mixture of arginine/leucine/lysine). Hence, ketone bodies (at least 3HB) should not be provided as nutraceuticals to everybody. Regarding therapy, this chapter further confirms that this specific AA mixture offers a promising treatment option to combat lipid and ketone body-associated cardiomyopathies.

Chapter 5 provides an overview and general discussion of our main findings. In particular, our novel findings that v-ATPase reassembly appears a suitable therapeutic target to rebalance cardiac substrate utilization in diabetic cardiomyopathy were discussed in more detail. In addition, an elaborated insight into strategies [i.e., short-term addition (1h) of this specific AA mixture at high concentration] to induce v-ATPase re-assembly in the diabetic heart was given, and possible promising targets [i.e., the identification of the proteins responsible for v-ATPase-mediated proton pumping, and the investigation of various signaling pathways, including Wnt, Notch, and AMPK, involved in v-ATPase function] to restore disturbances in energy metabolism were highlighted. Finally, given that v-ATPase integrates 
nutritional information in the heart, we also discuss possible upstream mechanisms regulating $\mathrm{v}$-ATPase function in response to nutrients in the heart.

In conclusion, the current thesis shows that v-ATPase integrates nutritional information in the heart. The main findings of this thesis lead to a deeper understanding of fundamental processes underlying diabetes, and will eventually lead to better possibilities for treating diabetic cardiomyopathy. 


\section{Samenvatting}

Het hart is een voortdurend actief orgaan dat voor zijn energievoorziening afhankelijk is van de continue aanvoer van brandstoffen. Onder normale, fysiologische omstandigheden is het hart een zogenoemde omnivoor, dat wil zeggen dat het allerlei brandstoffen als substraat voor de energievoorziening kan gebruiken. Dit wordt ook wel omschreven met de term 'metabool flexibel'. Kwantitatief zijn langketenige vetzuren en glucose de belangrijkste substraten. Daarnaast worden ook alternatieve substraten gebruikt, zoals aminozuren en ketonlichamen. Afhankelijk van fysiologische of pathofysiologische invloeden kan het aandeel van elk van deze substraten in de totale energievoorziening van het hart sterk wisselen.

In een serie recente studies is in ons lab aangetoond dat een enzym dat aanwezig is in bepaalde organellen van de hartspiercel (de endosomen) en daar verantwoordelijk is voor de zuurgraad van dit organel, een belangrijke rol speelt bij de keuze welk(e) substra(a)t(en) het hart gebruikt. Dit enzym is het vacuolar $\mathrm{H}^{+}$-ATPase of kortweg v-ATPase. Dit v-ATPase is een eiwitcomplex bestaande uit 14 eenheden, en is opgebouwd uit twee deel-complexen, namelijk subcomplex $\mathrm{V}_{0}$ dat zich in de membraan van het endosoom bevindt en het transport van $\mathrm{H}^{+}$ionen katalyseert, en subcomplex $\mathrm{V}_{1}$ dat in het cytosol aanwezig is en het enzymatische deel (ATPase) bevat dat zorgt voor de aandrijving van dit ionentransport. Het enzymcomplex is actief als beide subcomplexen zijn verbonden (assembly state) maar wordt inactief als het $\mathrm{V}_{1}$ subcomplex los laat en weg van het membraan het cytosol in migreert (disassembly state). Cycli van assembly en disassembly zijn snel en reversibel en maken het mogelijk dat de activiteit van het v-ATPase minutieus kan worden gereguleerd.

Als hartspiercellen worden blootgesteld aan bovenmatige hoeveelheden vetten (lipiden) of wanneer glucose als substraat wordt weggenomen blijkt het v-ATPase minder actief te worden (disassembly). Dit leidt dan tot een sterk verhoogde opname van vetzuren, zodanig dat vetzuren verreweg het belangrijkste substraat worden (>90\% van de energievoorziening). Deze bevinding ligt aan de basis van dit proefschrift. Het doel van de in dit proefschrift beschreven studies was te onderzoeken hoe v-ATPase reageert, direct of indirect, op veranderingen in het aanbod van de verschillende energierijke substraten (langketenige vetzuren, glucose, aminozuren en ketonlichamen). Meer specifiek is daarbij onderzocht of deze nieuwe kennis ingezet kan worden om lipide-geïnduceerde insuline resistentie en contractiele disfunctie van hartspiercellen te voorkomen danwel te behandelen. 
In hoofdstuk 1 wordt een inleiding gegeven over de opname en het gebruik van energierijke substraten door de hartspiercel, namelijk langketenige vetzuren (zoals palmitaat), glucose, aminozuren en ketonlichamen. Speciale aandacht is er voor de vetzuurtransporter CD36 en de glucosetransporter GLUT4, omdat die een belangrijke rol spelen bij het ontstaan van diabete cardiomyopathie. Dit hoofdstuk wordt afgesloten met een overzicht van de uitgevoerde studies.

Hoofdstuk 2 beschrijft een studie waarin bij zowel rattehartspiercellen als hartspiercellen van de mens is aangetoond dat een lipide-geïnduceerde remming van de vATPase activiteit kan worden tegengegaan door vergroting van de intracellulaire beschikbaarheid van glucose. Het onderliggend moleculaire mechanisme is uitgezocht en is als volgt: glucose herstelt de proton transport activiteit van het v-ATPase (toename assembly), waardoor de endosomen zuurder worden, er minder CD36 migreert van endosomen naar het sarcolemma. Vervolgens vermindert de vetzuuropname en daarmee de intracellulaire vetzuuropslag, waardoor de GLUT4-gemedieerde glucose opname niet meer wordt geblokkeerd (opheffen insuline resistentie) en uiteindelijk de contractie van de hartspiercellen normaliseert. Deze studie verschaft proof-of-principle dat beïnvloeding van de v-ATPase activiteit (via verandering in de assembly/disassembly status) een bruikbare aanpak is voor behandeling van lipide-geïnduceerde insuline resistentie en contractiele disfunctie van het hart en dus mogelijk voor diabete cardiomyopathie.

De opzet van de studie beschreven in Hoofdstuk 3 is analoog aan die van het voorgaande hoofdstuk maar is gericht op het effect van aminozuren, met name een mengsel van de aminozuren arginine, leucine en lysine. Dit aminozuurmengsel verhoogt de activiteit van het signaaleiwit mTOR, waarna dit bindt aan het $\mathrm{V}_{1}$ subcomplex van v-ATPase dat daardoor gemakkelijker bindt aan het $\mathrm{V}_{0}$ subcomplex en dan leidt tot een hogere $\mathrm{v}$-ATPase activiteit. Het was al bekend dat v-ATPase nodig is voor activatie van mTOR; het feit dat het omgekeerde ook opgaat, d.w.z. mTOR activatie leidt tot v-ATPase activatie, is een nieuwe bevinding. Aangetoond is dus dat mTOR en v-ATPase elkaar wederzijds activeren.

De toepasbaarheid van deze resultaten is groot. Immers, het hart zou op eenvoudige wijze beschermd kunnen worden tegen lipide-geïnduceerde insuline resistentie en contractiele disfunctie door het toedienen van een mengsel van bepaalde aminozuren. Omdat aminozuren een natuurlijk bestanddeel zijn van onze voeding, wordt een dergelijke toepassing wel aangeduid met de term 'nutraceutical'. 
Ook de studie in Hoofdstuk 4 is analoog opgezet aan die van de andere experimentele hoofdstukken. Hier is de blootstelling van hartspiercellen aan ketonlichamen bestudeerd. Het ketonlichaam 3- -hydroxyboterzuur (3HB) induceert disassembly van het v-ATPase, dus verlies van v-ATPase activiteit, uiteindelijk resulterend in vetstapeling en insuline resistentie. Deze nadelige effecten van 3HB, die geheel vergelijkbaar zijn met die van hoge concentraties lipiden, treden niet op bij blootstelling aan het ketonlichaam acetoacetaat. Ook bij deze studie kon het nadelige effect van $3 \mathrm{HB}$ op de v-ATPase activiteit worden tenietgedaan door toevoeging van het bovenbeschreven aminozuurmengsel arginine/leucine/lysine, en kon zodoende het ontstaan van insuline resistentie worden voorkomen.

De resultaten van de verschillende studies worden in Hoofdstuk 5 samengevat, geïntegreerd en in detail bediscussieerd. Het endosomale enzym v-ATPase blijkt een sleutelrol te vervullen bij het ontstaan van voedingsgerelateerde insuline resistentie van hartspiercellen en diabete cardiomyopathie. Op moleculair niveau ligt de cyclus van assembly en disassembly van de subcomplexen $\mathrm{V}_{0}$ en $\mathrm{V}_{1}$ hieraan ten grondslag. Tevens is aangetoond dat de activiteit van v-ATPase kan worden gemanipuleerd door variatie van de aanwezigheid van de vier onderzochte energierijke substraten (langketenige vetzuren, glucose, ketonlichamen en aminozuren). Een dergelijke manipulatie van de v-ATPase activiteit kan naar verwachting worden ingezet als metabole therapie ter voorkoming en behandeling van diabete cardiomyopathie, en wellicht ook van andere metabole hartziekten zoals pathologische hypertrofie (dat gepaard gaat met een preferentieel glucoseverbruik door het hart). De ontdekking dat v-ATPase reageert op veranderingen in de aanvoer van energierijke substraten, dus in feite op veranderingen in voedingssamenstelling, zou ook in bredere zin moeten worden bestudeerd. Te denken valt aan het effect op het functioneren van andere weefsels, met name de skeletspier.

Tot besluit kan gesteld worden dat de in dit proefschift beschreven experimentele studies hebben geleid tot de identificatie in de hartspier van het endosomale enzym v-ATPase als centraal punt waar invloeden van verschillende voedingscomponenten samenkomen. Omdat tevens de betrokken moleculaire mechanismen grotendeels zijn opgehelderd, kan gerichte therapie worden opgezet ter bestrijding van cardiale insulineresistentie en uiteindelijk diabete cardiomyopathie. 


\section{总结}

在正常生理情况下, 心脏作为一个灵活性的代谢器官, 可以利用多种能源底物为 机体提供能量补给, 因此心脏被视为杂食性器官。为了满足机体的能量需求, 健康成年 人的心脏主要以长链脂肪酸 (FA) 和葡萄糖能量补给为主, 然而, 在其他生理或者病理 条件下, 心脏的能量底物也来自其他物质, 如氨基酸 (AA) 和酮体。我们实验室最新研 究表明, vacuolar $H^{+}$-ATPase ( $v$-ATPase) 复合型蛋白与心脏能量代谢调控密切相关。 V-ATPase 是一种多聚体蛋白质复合物（>14 个亚基），在结构上分为胞质 $\mathrm{V}_{1}$ 区域和跨 膜 $V_{0}$ 区域物, 它们分别具有催化 ATP 活性和维持胞内体酸化的旋转机制。值得我们注 意的是, $V_{1}$ 和 $V_{0}$ 亚复合物的组装/分解周期是快速且可逆的过程, 因此可以快速调节 $v-$ ATPase 功能。如我们研究发现, 当心肌细胞培养于高脂或无葡萄糖的培养环境中, $\mathrm{v}^{-}$ ATPase 的两个亚复合 $\left(V_{1} / V_{0}\right)$ 将会分解, 进一步导致 v-ATPase 功能丢失。基于这些发 现, 该论文旨在研究心脏能量底物 [如, 脂质（棕㭣酸）, 葡萄糖, 氨基酸 (AA) 和酮 体] 对 v-ATPase 功能的直接/间接影响。此外, 我们也探究是否 v-ATPase 功能的增加 会改善高脂诱导的胰岛素拮抗及心肌收缩功能, 且揭示潜在的分子机制。

第 1 章简约概述了 (啮齿动物/人) 心肌细胞对能量底物, 如脂质（棕榈酸）, 葡 萄糖, 氨基酸 ( $\mathrm{AA}$ ) 和酮体的代谢及利用。此外, 本章也阐述了脂肪酸转运蛋白 (CD36) 和葡萄糖转运蛋白 (GLUT4) 在心脏的脂代谢中的主要作用, 且探讨了导致糖尿病性心 肌病发生的潜在机制, 尤其是高脂介导 $\mathrm{v}$-ATPase 功能异常的代谢通路。在本章的最后 部分, 我们也详细讨论了该论文的主要目的和概述。

第 2 章揭示, 在啮齿动物/人心肌细胞中, 葡萄糖代谢利用率增加可以改善高脂介 导 v-ATPase 的功能异常。具体而言, v-ATPase 功能的恢复会促进脂质超载（啮齿动物 / 人) 心肌细胞的胞内体酸化, 进一步抑制 CD36 参与调节的脂质蓄积, 进而改善胰岛素 刺激的葡萄糖代谢以及心肌收缩功能。基于这些研究发现, 本章确立了这一潜在策略/ 方案: 增加 v-ATPase 功能能够改善高脂介导的胰岛素拮抗和心肌收缩功能障碍。

第 3 章研究结果揭示, 在（啮齿动物/人）心肌细胞中, 补充氨基酸混合物（AA, 精氨酸/亮氨酸/赖氨酸）能够激活 mTOR, 然后激活的 mTOR 与 $\mathrm{v}-\mathrm{ATP} a \mathrm{se}$ 的 $\mathrm{V}_{1}$ 亚复合物 结合, 从而促进 $V_{1} / V_{0}$ 区域的重新组装。鉴于 $v_{-}$-ATPase 是 mTOR 激活的主要支架之一, 本章也首次提出 mTOR 的激活也能调节 $\mathrm{v}$-ATPase 功能, 这证实 $\mathrm{v}-A T P a s e$ 和 mTOR 的互作 是双向的。此外, 该氨基酸 ( AA) 激活的 mTOR - v-ATPase 轴也能够参与胞内体酸化的 
调节, 从而抑制 CD36 介导的脂肪酸吸收, 进一步保护心肌细胞免于高脂所引起的胰岛 素抵抗以及收缩功能障碍。因此, 本章中的研究结果表明, 氨基酸调控的 v-ATPase 重 组可能为高脂诱导的心肌疾病提供了简单有效的治疗方案。

第 4 章阐述了，啮齿动物/人的心肌细胞长期暴露于高剂量酮体，如 3- $\beta$-羟基丁 酸酯（3HB）, 会导致 $v$-ATPase 结构分解, 进而引起 $v$-ATPase 功能丢失。与高脂介导 的 $v$-ATPase 分子机制类似, 3- $\beta$-羟基丁酸酯（3HB）诱导的 $v-A T P a s e$ 功能降低也会引 起胞内体碱性化, 随后刺激脂肪酸转运蛋白（CD36）易位至细胞肌膜, 肌细胞脂质进一 步蓄积, 最后引起胰岛素拮抗以及心肌收缩功能障碍。值得注意的是, 该章也进一步证 实补充氨基酸混合物（AA，精氨酸/亮氨酸/赖氨酸）能够有效改善由高酮/高脂所引起 的胰岛素拮抗以及心肌收缩功能障碍。因此, 基于该章节的研究发现, 酮体日粮/饮料, 3 - $\beta$-羟基丁酸酯（3HB），不应作为保健食品提供给所有人群。此外，关于这方面的治 疗方案, 本章也进一步证实氨基酸混合物（AA，精氨酸/亮氨酸/赖氨酸）为高脂/高酮 的相关心脏疾病提供了有效的治疗选择。

第 5 章详细地讨论了当前的新发现, 即重组 v-ATPase 是一个调节糖尿病性心肌疾 病能量代谢的治疗靶标。此外, 该章也介绍了其他调控糖尿病性心肌细胞中 $\mathrm{v}-\mathrm{ATPase}$ 重组的策略, 例如, 高浓度短期补充 ( $1 \mathrm{~h}$ ) 氨基酸混合物 ( AA, 精氨酸/亮氨酸/赖氨酸), 同时, 也提出了其他的潜在靶标 [如, $v$-ATPase 介导的质子泵蛋白, 以及涉及 $v-A T P a s e$ 功能的各种信号通路 (包括 Wnt, Notch 和 AMPK) ] 参与调控改善心肌能量代谢的紊乱。 最后, 鉴于 $\mathrm{v}^{-}$ATPase 能够整合了心脏中营养信息的研究发现, 我们也讨论了 ${ }^{\mathrm{v}}$-ATPase 功能的调节以及以响应心脏细胞营养代谢的上游机制。

总之, 当前研究结果表明, $v-A T P a s e$ 整合了心脏中的营养信息。本论文的主要发 现使人们对糖尿病预防及治疗的基本分子机制有了较为深入的理解, 最终将为治疗糖尿 病性心肌疾病提供更好的方案及靶点。 